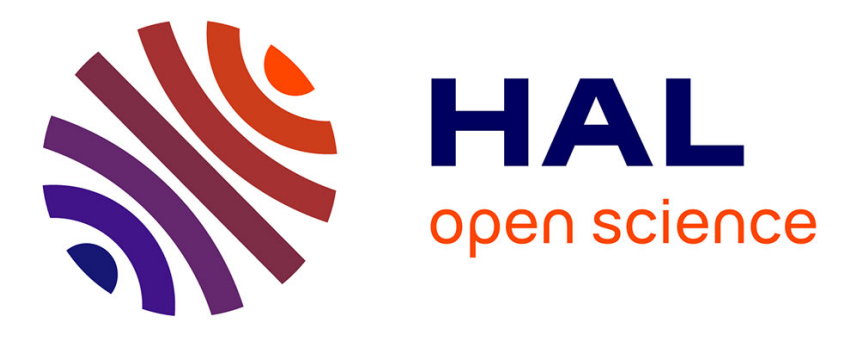

\title{
Self-Interference in Full-Duplex Multi-User MIMO Channels
}

Arman Shojaeifard, Kai-Kit Wong, Marco Di Renzo, Gan Zheng, Khairi Ashour Hamdi, Jie Tang

\section{To cite this version:}

Arman Shojaeifard, Kai-Kit Wong, Marco Di Renzo, Gan Zheng, Khairi Ashour Hamdi, et al.. SelfInterference in Full-Duplex Multi-User MIMO Channels. IEEE Communications Letters, 2017, 21 (4), pp.841 - 844. 10.1109/LCOMM.2016.2647709 . hal-01880021

\section{HAL Id: hal-01880021 https://hal.science/hal-01880021}

Submitted on 7 Jul 2020

HAL is a multi-disciplinary open access archive for the deposit and dissemination of scientific research documents, whether they are published or not. The documents may come from teaching and research institutions in France or abroad, or from public or private research centers.
L'archive ouverte pluridisciplinaire HAL, est destinée au dépôt et à la diffusion de documents scientifiques de niveau recherche, publiés ou non, émanant des établissements d'enseignement et de recherche français ou étrangers, des laboratoires publics ou privés.

\section{(c)(1)}

Distributed under a Creative Commons Attribution| 4.0 International License 


\title{
Self-Interference in Full-Duplex Multi-User MIMO Channels
}

\author{
Arman Shojaeifard, Member, IEEE, Kai-Kit Wong, Fellow, IEEE, \\ Marco Di Renzo, Senior Member, IEEE, Gan Zheng, Senior Member, IEEE, \\ Khairi Ashour Hamdi, Senior Member, IEEE, and Jie Tang, Member, IEEE
}

\begin{abstract}
We consider a multi-user multiple-input multipleoutput setup, where full-duplex multi-antenna nodes apply linear beamformers to simultaneously transmit and receive multiple streams over Rician fading channels. The exact first and second positive moments of the residual self-interference (SI), involving the squared norm of a sum of non-identically distributed random variables, are derived in closed-form. The method of moments is hence invoked to provide a Gamma approximation for the residual SI distribution. The proposed theorem holds under arbitrary linear precoder/decoder design, antenna array size, number of information streams, and SI cancellation capability.
\end{abstract}

Index Terms-Multiple-input multiple-output (MIMO), full-duplex (FD), self-interference (SI), Rician fading model.

\section{INTRODUCTION}

$\mathbf{T}$ O DATE, wireless systems, have been designed and dimensioned under a complete separation of the transmit and receive functions, a.k.a., half-duplex (HD) mode. This is typically achieved via orthogonal radio frequency (RF) partitioning, e.g., in time-division duplex (TDD) and frequencydivision duplex (FDD) systems. The motivation behind this trend has been to avoid the overwhelming self-interference (SI) arising from the bi-directional operation.

The rapid increase in traffic, under limited RF resources, however, has driven the incentive for an overhaul in the wireless system design. A candidate solution involves transceiving information over the same RF resources, i.e., full-duplex (FD) mode [1]. There is a growing surge of interest in this topic, as in theory, FD has the potential to double the spectral efficiency versus HD. Several point-to-point prototypes of FD radio nodes have been shown in practice recently [2].

Despite pioneering efforts, FD operation in the context of multi-user multiple-input multiple-output (MIMO) setups is not well understood. A long-standing limitation is in the modeling of the residual SI using the Rayleigh distribution [3].

Manuscript received November 24, 2016; revised December 27, 2016; accepted December 30, 2016. Date of publication January 4, 2017; date of current version April 7, 2017. This work was supported by the Engineering and Physical Sciences Research Council (EPSRC) under grants EP/N008219/1 and EP/N007840/1. The associate editor coordinating the review of this letter and approving it for publication was Y. Deng.

A. Shojaeifard and K.-K. Wong are with the Department of Electronic and Electrical Engineering, University College London, London, U.K. (e-mail: a.shojaeifard@ucl.ac.uk; kai-kit.wong@ucl.ac.uk).

M. Di Renzo is with the Laboratoire des Signaux et Systèmes, CNRS, CentraleSupélec, Univ Paris Sud, Université Paris-Saclay, Gif-sur-Yvette, France (e-mail: marco.direnzo@12s.centralesupelec.fr).

G. Zheng is with the Wolfson School of Mechanical, Electrical and Manufacturing Engineering, Loughborough University, Loughborough, U.K. (e-mail: g.zheng@lboro.ac.uk).

K. A. Hamdi is with the School of Electrical and Electronic Engineering, The University of Manchester, Manchester, U.K. (e-mail: k.hamdi@manchester.ac.uk).

J. Tang is with the School of Electronic and Information Engineering, South China University of Technology, Guangzhou, China (e-mail: eejtang@scut.edu.cn).

Digital Object Identifier 10.1109/LCOMM.2016.2647709
This approach, whilst improving on the common perfect SI cancellation assumption, differs from measurements showing the residual SI channel undergoes Rician fading [1]. The residual SI distribution over Rician fading channels is thus required for facilitating performance analysis and optimization.

In this Letter, we consider a FD multi-antenna node communicating with multiple FD radios over the same RF resources. The Rician fading model is employed to capture the residual SI under arbitrary cancellation through tuning of the distribution parameters by design or measurements. With linear processing, it is not feasible to derive the statistics of the residual SI channel power gain directly. Here, exact closed-form expressions for the residual SI first and second positive moments are developed. We then exploit the method of moments in order to obtain an explicit Gamma approximation for the distribution of the residual SI over FD multi-user MIMO Rician fading channels. The validity of the theoretical findings is confirmed through MonteCarlo simulations of the entire RF transmit/receive processing chain.

Notation: $\boldsymbol{X}$ is a matrix with $(n, m)$-th entry $\{\boldsymbol{X}\}_{n, m} ; \boldsymbol{x}$ is a vector with $k$-th element $\{\boldsymbol{x}\}_{k} ; T$, $\dagger$, and + are the transpose, Hermitian-transpose, and pseudo-inverse; $\mathbb{E}\{$.$\} is the expected$ value; $\mathbb{V}\{$.$\} is the variance; \mathcal{P}($.$) is the probability density$ function (pdf); $|$.$| is the modulus; \|$.$\| is the norm; and I_{0}($.$) is$ the zeroth-order Bessel function of the first kind, respectively.

\section{SySTEM MODEL}

Consider $L$ cells, where in each cell $l, l \in \mathcal{L}=\{1, \ldots, L\}$, a FD multi-antenna node $l_{0}$ communicates with respect to multiple FD radios $l_{k}, k \in \mathcal{K}=\{1, \ldots, K\}$. Let $M$ and $N$ respectively denote the number of transmit and receive antennas at the FD multi-antenna nodes. The FD radios are assumed to be equipped with two antennas, one for transmission, and the other for reception. An application example is FD multi-antenna base stations communicating in the downlink/uplink with multiple FD mobile terminals. Assuming $K \leq \min (N, M)$, channel assignment is not required.

Let $\boldsymbol{h}_{\left[l_{k}, j_{0}\right]} \in \mathcal{C}^{1 \times M}$ and $\boldsymbol{h}_{\left[j_{0}, l_{k}\right]} \in \mathcal{C}^{N \times 1}$ respectively denote the transmit and receive channels between the FD multiantenna node in cell $j$ and the $k$-th FD radio in cell $l$. The respective combined channels are $\boldsymbol{H}_{\left[l_{K}, j_{0}\right]}=\left[\boldsymbol{h}_{\left[l_{k}, j_{0}\right]}^{T}\right]_{1 \leq k \leq K}^{T} \in$ $\mathcal{C}^{K \times M}$ and $\boldsymbol{H}_{\left[j_{0}, l_{K}\right]}=\left[\boldsymbol{h}_{\left[j_{0}, l_{k}\right]}\right]_{1 \leq k \leq K} \in \mathcal{C}^{N \times K}$. The crossmode channels between $l_{0}$ and $j_{0}$, and between $l_{k}$ and $j_{k}$, are $\boldsymbol{H}_{\left[j_{0}, l_{0}\right]} \in \mathcal{C}^{N \times M}$, and $h_{\left[j_{k}, l_{k}\right]}$, respectively. The residual SI channels are Rician distributed with elements drawn from the complex Gaussian distribution $\mathcal{C N}\left(\mu, v^{2}\right)$ [4], [5]. Other channels are subject to Rayleigh fading with elements drawn from $\mathcal{C N}(0,1)$. Perfect channel state information (CSI) is assumed to be available.

We consider arbitrary linear beamforming design at the FD multi-antenna nodes. Let $\boldsymbol{s}_{\left[l_{K}, l_{0}\right]}=\left[s_{\left[l_{k}, l_{0}\right]}\right]_{1 \leq k \leq K}^{T} \in \mathcal{C}^{K \times 1}$, 


$$
\begin{aligned}
& y_{\left[l_{k}, l_{0}\right]}=\underbrace{\boldsymbol{h}_{\left[l_{k}, l_{0}\right]} \boldsymbol{v}_{\left[l_{k}, l_{0}\right]} S_{\left[l_{k}, l_{0}\right]}}_{\text {useful signal, } x_{\left[l_{k}, l_{0}\right]}}+\underbrace{\boldsymbol{h}_{\left[l_{k}, l_{0}\right]} \sum_{u \in \mathcal{K} \backslash\{k\}} \boldsymbol{v}_{\left[l_{u}, l_{0}\right]} s_{\left[l_{u}, l_{0}\right]}}_{\text {multi-user interference, } m u i_{\left[l_{k}, l_{0}\right]}}+\underbrace{\sum_{j \in \mathcal{L} \backslash\{l\}} \boldsymbol{h}_{\left[l_{k}, j_{0}\right]} \boldsymbol{V}_{\left[j_{K}, j_{0}\right]} \boldsymbol{s}_{\left[j_{K}, j_{0}\right]}}_{\text {inter-cell interference, } \text { ici }_{\left[l_{k}, l_{0}\right]}} \\
& +\underbrace{\sum_{j \in \mathcal{L}, u \in \mathcal{K} \backslash\{l, k\}} h_{\left[l_{k}, j_{u}\right]} S_{\left[j_{0}, j_{u}\right]}}_{\text {cross-mode interference, } c m i i_{\left[l_{k}, l_{0}\right]}}+\underbrace{h_{\left[l_{k}, l_{k}\right]} S_{\left[l_{0}, l_{k}\right]}}_{\text {residual self-interference, } s i_{\left[l_{k}, l_{0}\right]}}+\underbrace{\eta_{\left[l_{k}, l_{0}\right]}}_{\text {noise, } n_{\left[l_{k}, l_{0}\right]}} \\
& y_{\left[l_{0}, l_{k}\right]}=\underbrace{\boldsymbol{w}_{\left[l_{0}, l_{k}\right]}^{T} \boldsymbol{h}_{\left[l_{0}, l_{k}\right]} s_{\left[l_{0}, l_{k}\right]}}_{\text {useful signal, } x_{\left[l_{0}, l_{k}\right]}}+\underbrace{\boldsymbol{w}_{\left[l_{0}, l_{k}\right]}^{T} \sum_{u \in \mathcal{K} \backslash\{k\}} \boldsymbol{h}_{\left[l_{0}, l_{u}\right]} S_{\left[l_{0}, l_{u}\right]}}_{\text {multi-user interference, } \text { mui }_{\left[l_{0}, l_{k}\right]}}+\underbrace{\boldsymbol{w}_{\left[l_{0}, l_{k}\right]}^{T} \sum_{j \in \mathcal{L}, u \in \mathcal{K} \backslash\{l, k\}} \boldsymbol{h}_{\left[l_{0}, j_{u}\right]} S_{\left[j_{0}, j_{u}\right]}}_{\text {inter-cell interference, } i_{i} i_{\left[l_{0}, l_{k}\right]}} \\
& +\underbrace{\boldsymbol{w}_{\left[l_{0}, l_{k}\right]}^{T} \sum_{j \in \mathcal{L} \backslash\{l\}} \boldsymbol{H}_{\left[l_{0}, j_{0}\right]} \boldsymbol{V}_{\left[j_{K}, j_{0}\right]} \boldsymbol{s}_{\left[j_{K}, j_{0}\right]}}+\underbrace{\boldsymbol{w}_{\left[l_{0}, l_{k}\right]}^{T} \boldsymbol{H}_{\left[l_{0}, l_{0}\right]} \boldsymbol{V}_{\left[l_{K}, l_{0}\right]} \boldsymbol{s}_{\left[l_{K}, l_{0}\right]}}_{\text {residual self-interference, } s i_{\left[l_{0}, l_{k}\right]}}+\underbrace{\boldsymbol{w}_{\left[l_{0}, l_{k}\right]}^{T} \boldsymbol{\eta}_{\left[l_{0}, l_{K}\right]}}_{\text {scaled noise, } n_{\left[l_{0}, l_{k}\right]}} \\
& \text { cross-mode interference, } \mathrm{cmi}_{\left[l_{0}, l_{k}\right]}
\end{aligned}
$$

$\mathbb{E}\left\{\left|s_{\left[l_{k}, l_{0}\right]}\right|^{2}\right\}=1$, denote the complex information vector from $l_{0}$ to all $l_{k}$. The corresponding complex information vector in the reverse communications direction is $\boldsymbol{s}_{\left[l_{0}, l_{K}\right]}=$ $\left[s_{\left[l_{0}, l_{k}\right]}\right]_{1 \leq k \leq K}^{T} \in \mathcal{C}^{K \times 1}, \mathbb{E}\left\{\left|s_{\left[l_{0}, l_{k}\right]}\right|^{2}\right\}=1$. The transmit signal vector under linear precoding at $l_{0}$ is hence constructed as $\boldsymbol{t}_{\left[l_{K}, l_{0}\right]}=\boldsymbol{V}_{\left[l_{K}, l_{0}\right]} \boldsymbol{s}_{\left[l_{K}, l_{0}\right]}$ where $\boldsymbol{V}_{\left[l_{K}, l_{0}\right]}=\left[\boldsymbol{v}_{\left[l_{k}, l_{0}\right]}\right]_{1 \leq k \leq K} \in$ $\mathcal{C}^{M \times K}$ is the precoding matrix. In addition, the linear receive filter at $l_{0}$ is defined as $\boldsymbol{W}_{\left[l_{0}, l_{K}\right]}=\left[\boldsymbol{w}_{\left[l_{0}, l_{k}\right]}^{T}\right]_{1 \leq k \leq K}^{T} \in \mathcal{C}^{K \times N}$.

The received signal from the FD multi-antenna node $l_{0}$ at its FD radio $l_{k}$ is expressed as in (1), as shown at the top of this page, where $\eta_{\left[l_{k}, l_{0}\right]}$ is the complex additive white Gaussian noise (AWGN). The post-processing received signal in the reverse communications direction is written as in (2), as shown at the top of this page, where $\eta_{\left[l_{0}, l_{K}\right]} \in \mathcal{C}^{N \times 1}$ is the circularlysymmetric complex AWGN vector.

\section{Signals Statistics}

Next, we formulate the signal-to-interference-plus-noise ratios (SINRs). Note $d$ and $u$ are respectively used in place of $\left[l_{k}, l_{0}\right]$ and $\left[l_{0}, l_{k}\right]$ where the context is clear. Hence,

$$
\mathcal{Y}_{y_{d}}=\frac{\mathcal{X}_{x_{d}}}{\mathcal{I}_{\text {mui }_{d}}+\mathcal{I}_{i c i_{d}}+\mathcal{I}_{c m i_{d}}+\mathcal{I}_{s i_{d}}+\mathcal{N}_{n_{d}}}
$$

where we have $\mathcal{X}_{x_{d}}=\left|\boldsymbol{h}_{\left[l_{k}, l_{0}\right]} \boldsymbol{v}_{\left[l_{k}, l_{0}\right]}\right|^{2}, \mathcal{I}_{m u i_{d}}=\sum_{u \in \mathcal{K} \backslash\{k\}}$ $\left|\boldsymbol{h}_{\left[l_{k}, l_{0}\right]} \boldsymbol{v}_{\left[l_{u}, l_{0}\right]}\right|^{2}, \mathcal{I}_{i c i_{d}}=\sum_{j \in \mathcal{L} \backslash\{l\}}\left\|\boldsymbol{h}_{\left[l_{k}, j_{0}\right]} \boldsymbol{V}_{\left[j_{K}, j_{0}\right]}\right\|^{2}, \mathcal{I}_{c m i_{d}}=$ $\sum_{j \in \mathcal{L}, u \in \mathcal{K} \backslash\{l, k\}}\left|h_{\left[l_{k}, j_{u}\right]}\right|^{2}, \quad \mathcal{I}_{s i_{d}}=\left|h_{\left[l_{k}, l_{k}\right]}\right|^{2}$, and $\mathcal{N}_{n_{d}}=$ $\left|\eta_{\left[l_{k}, l_{0}\right]}\right|^{2}$ respectively. Moreover,

$$
\mathcal{Y}_{y_{u}}=\frac{\mathcal{X}_{x_{u}}}{\mathcal{I}_{m u i_{u}}+\mathcal{I}_{i c i_{u}}+\mathcal{I}_{c m i_{u}}+\mathcal{I}_{s i_{u}}+\mathcal{N}_{n_{u}}}
$$

with the signals $\mathcal{X}_{x_{u}}=\left|\boldsymbol{w}_{\left[l_{0}, l_{k}\right]}^{T} \boldsymbol{h}_{\left[l_{0}, l_{k}\right]}\right|^{2}, \mathcal{I}_{m u i_{u}}=\sum_{u \in \mathcal{K} \backslash\{k\}}$ $\left|\boldsymbol{w}_{\left[l_{0}, l_{k}\right]}^{T} \boldsymbol{h}_{\left[l_{0}, l_{u}\right]}\right|^{2}, \quad \mathcal{I}_{i c i_{u}}=\sum_{j \in \mathcal{L}, u \in \mathcal{K} \backslash\{l, k\}}\left|\boldsymbol{w}_{\left[l_{0}, l_{k}\right]}^{T} \boldsymbol{h}_{\left[l_{0}, j_{u}\right]}\right|^{2}$, $\mathcal{I}_{c m i_{u}}=\sum_{j \in \mathcal{L} \backslash\{l\}}\left\|\boldsymbol{w}_{\left[l_{0}, l_{k}\right]}^{T} \boldsymbol{H}_{\left[l_{0}, j_{0}\right]} \boldsymbol{V}_{\left[j_{K}, j_{0}\right]}\right\|^{2}, \mathcal{I}_{s i_{u}}=\| \boldsymbol{w}_{\left[l_{0}, l_{k}\right]}^{T}$ $\boldsymbol{H}_{\left[l_{0}, l_{0}\right]} \boldsymbol{V}_{\left[l_{K}, l_{0}\right]} \|^{2}$, and $\mathcal{N}_{n_{u}}=\left|\boldsymbol{w}_{\left[l_{0}, l_{k}\right]}^{T} \boldsymbol{\eta}_{\left[l_{0}, l_{K}\right]}\right|^{2}$.

With linear processing over isotropic MIMO Rayleigh fading channels, the signals can be captured using the Gamma distribution [6]. For example, consider linear zeroforcing $(\mathrm{ZF})$ for removing multi-user interference by setting (i) the column vectors of $\boldsymbol{V}_{\left[l_{K}, l_{0}\right]}$ equal to the normalized columns of $\boldsymbol{H}_{\left[l_{K}, l_{0}\right]}^{+}=\boldsymbol{H}_{\left[l_{K}, l_{0}\right]}^{\dagger}\left(\boldsymbol{H}_{\left[l_{K}, l_{0}\right]} \boldsymbol{H}_{\left[l_{K}, l_{0}\right]}^{\dagger}\right)^{-1}$, and (ii) the row vectors of $\boldsymbol{W}_{\left[l_{0}, l_{K}\right]}$ equal to the normalized rows of $\boldsymbol{H}_{\left[l_{0}, l_{K}\right]}^{+}=\left(\boldsymbol{H}_{\left[l_{0}, l_{K}\right]}^{\dagger} \boldsymbol{H}_{\left[l_{0}, l_{K}\right]}\right)^{-1} \boldsymbol{H}_{\left[l_{0}, l_{K}\right]}^{\dagger}$. With the projection of each useful channel vector onto the nullspace spanned by the multi-user interference, we have $\left|\boldsymbol{h}_{\left[l_{k}, l_{0}\right]} \boldsymbol{v}_{\left[l_{k}, l_{0}\right]}\right|^{2} \sim$ $\operatorname{Gamma}(M-K+1,1)$ and $\left|\boldsymbol{w}_{\left[l_{0}, l_{k}\right]}^{T} \boldsymbol{h}_{\left[l_{0}, l_{k}\right]}\right|^{2} \sim \operatorname{Gamma}(N-$ $K+1,1)$. Moreover, $\left|\boldsymbol{w}_{\left[l_{0}, l_{k}\right]}^{T} \boldsymbol{h}_{\left[l_{0}, j_{u}\right]}\right|^{2} \sim \operatorname{Gamma}(1,1)$ and $\left|h_{\left[l_{k}, j_{u}\right]}\right|^{2} \sim \operatorname{Gamma}(1,1)$. Further, under the assumption that the outer-cell precoding vectors are independent, we have $\left\|\boldsymbol{h}_{\left[l_{k}, j_{0}\right]} \boldsymbol{V}_{\left[j_{K}, j_{0}\right]}\right\|^{2} \sim \operatorname{Gamma}(U, 1)$ and $\left\|\boldsymbol{w}_{\left[l_{0}, l_{k}\right]}^{T} \boldsymbol{H}_{\left[l_{0}, j_{0}\right]} \boldsymbol{V}_{\left[j_{K}, j_{0}\right]}\right\|^{2} \sim \operatorname{Gamma}(U, 1)$.

Next, we consider the residual SI channel of an arbitrary FD radio $l_{k}$, with single transmit and receive antennas, $h_{\left[l_{k}, l_{0}\right]} \sim \mathcal{C N}\left(\mu, v^{2}\right)$. The corresponding residual SI channel power gain, $\mathcal{I}_{s i_{d}}=\left|h_{\left[l_{k}, l_{0}\right]}\right|^{2}$, has a non-central Chi-squared distribution

$$
\mathcal{P}_{\mathcal{I}_{s i}}(x)=\frac{1+\varpi}{\Omega} \exp \left(-\left(\varpi+\frac{(1+\varpi) x}{\Omega}\right)\right) I_{0}\left(2 \sqrt{\frac{\varpi(1+\varpi) x}{\Omega}}\right)
$$

where $\varpi$ and $\Omega$ are the Rician factor and fading attenuation with $\mu \triangleq \sqrt{\frac{\varpi \Omega}{\varpi+1}}$ and $v \triangleq \sqrt{\frac{\Omega}{\varpi+1}}$, respectively. The method of moments can be applied to provide a Gamma approximation for the residual SI in (5). Hence, the residual SI channel power gain of an arbitrary FD radio $k$, with single transmit and receive antennas, and fading coefficients drawn from $\mathcal{C N}\left(\mu, v^{2}\right)$, is approximated using $\mathcal{I}_{s i_{d}}=\left|h_{\left[l_{k}, l_{0}\right]}\right|^{2} \sim$ $\operatorname{Gamma}(\kappa, \theta)$ where

$$
\kappa \triangleq \frac{\left(\mu^{2}+v^{2}\right)^{2}}{\left(2 \mu^{2}+v^{2}\right) v^{2}}
$$

and

$$
\theta \triangleq \frac{\left(2 \mu^{2}+v^{2}\right) v^{2}}{\mu^{2}+v^{2}}
$$

The residual SI channel power gain at the FD multi-antenna nodes, under arbitrary number of antennas and information streams, involves the squared norm of a sum of non-identically distributed random variables. For instance, for the decoding of the transmitted signal from the arbitrary FD node $l_{k}$ at the receiver of the FD multi-antenna node $l_{0}$, we have $\mathcal{I}_{s i_{u}}=$ $\left\|\boldsymbol{w}_{\left[l_{0}, l_{k}\right]}^{T} \boldsymbol{H}_{\left[l_{0}, l_{0}\right]} \boldsymbol{V}_{\left[l_{K}, l_{0}\right]}\right\|^{2} \quad$ where $\boldsymbol{w}_{\left[l_{0}, l_{k}\right]}^{T} \boldsymbol{H}_{\left[l_{0}, l_{0}\right]} \boldsymbol{V}_{\left[l_{K}, l_{0}\right]}=$ $\left[\sum_{n=1}^{N} \sum_{m=1}^{M}\left\{\boldsymbol{w}_{\left[l_{0}, l_{k}\right]}^{T}\right\}_{n}\left\{\boldsymbol{H}_{\left[l_{0}, l_{0}\right]}\right\}_{n, m}\left\{\boldsymbol{V}_{\left[l_{K}, l_{0}\right]}\right\}_{m, k}\right]_{1 \leq k \leq K}$. 


$$
\begin{aligned}
& \kappa \triangleq \frac{K(N+1)(M-K+2)\left(\mu^{2}+v^{2}\right)^{2}}{\left(2 N M+\frac{K(M-K+2)}{(M+1)}(N M-N-M-1)\right) \mu^{4}+(N+1)(M+1) v^{2}\left(2 \mu^{2}+v^{2}\right)} \\
& \theta \triangleq \frac{\left(2 N M+\frac{K(M-K+2)}{(M+1)}(N M-N-M-1)\right) \mu^{4}+(N+1)(M+1) v^{2}\left(2 \mu^{2}+v^{2}\right)}{(N+1)(M-K+2)\left(\mu^{2}+v^{2}\right)}
\end{aligned}
$$

TABLE I

Residual Si Distribution Using Gamma Moment Matching in Some Special Cases of Interest

\begin{tabular}{|c|c|c|}
\hline Single-User & $\kappa=\frac{(N+1)(M+1)\left(\mu^{2}+\nu^{2}\right)^{2}}{(3 N M-N-M-1) \mu^{4}+2(N+1)(M+1) \mu^{2} \nu^{2}+(N+1)(M+1) \nu^{4}}$ & $\theta=\mu^{2}+\nu^{2}+\frac{2(M N-N-M-1) \mu^{4}}{(N+1)(M+1)\left(\mu^{2}+\nu^{2}\right)}$ \\
\hline Rayleigh Channel & $\kappa=\frac{K(\max (N, M)-K+2)}{\max (N, M)+1}$ & $\theta=\frac{\max (N, M)+1}{\max (N, M)-K+2}$ \\
\hline Massive MIMO & $\kappa=\frac{K\left(\mu^{2}+\nu^{2}\right)^{2}}{(K+2) \mu^{4}+2 \mu^{2} \nu^{2}+\nu^{4}}$ & $\theta=\frac{(K+2) \mu^{4}+2 \mu^{2} \nu^{2}+\nu^{4}}{\mu^{2}+\nu^{2}}$ \\
\hline
\end{tabular}

It is therefore not possible to directly derive the exact pdf of $\mathcal{I}_{s i_{u}}$. As a result, we derive closed-form expressions for the exact first and second positive moments of $\mathcal{I}_{s i_{u}}$. We then apply the method of moments in order to develop a unified closed-form approximation for the residual SI distribution over multi-user MIMO Rician fading channels.

Theorem: Consider the residual SI fading channel of an arbitrary FD multi-antenna node $l_{0}$ with $M$ transmit and $N$ receive antennas, $\boldsymbol{H}_{\left[l_{0}, l_{0}\right]}$, comprising elements distributed according to $\mathcal{C N}\left(\mu, v^{2}\right)$. Using a linear precoder $\boldsymbol{V}_{\left[l_{K}, l_{0}\right]}$ and a linear decoder $\boldsymbol{W}_{\left[l_{0}, l_{K}\right]}, l_{0}$ simultaneously transmits and receives information streams with respect to $K F D$ radios, each equipped with single transmit and receive antennas. The corresponding residual SI channel power gain at the $F D$ multi-antenna node $l_{0}$ can be approximated using $\mathcal{I}_{s i_{u}}=$ $\left\|\boldsymbol{w}_{\left[l_{0}, l_{k}\right]}^{T} \boldsymbol{H}_{\left[l_{0}, l_{0}\right]} \boldsymbol{V}_{\left[l_{K}, l_{0}\right]}\right\|^{2} \sim \operatorname{Gamma}(\kappa, \theta)$ where (8) and (9), as shown at the top of this page.

Proof: See Appendix.

The unified expression in the Theorem holds for arbitrary linear precoder/decoder design, number of transmit/receive antennas, number of information streams, and Rician channel statistics (and in turn arbitrary SI cancellation capability). The distribution of the residual SI in special scenarios of interest can also be readily obtained in closed-form. Some useful examples are provided for the sake of demonstration in the Table.

\section{Performance Analysis}

Here, we perform Monte-Carlo of the entire RF transmit/ receive processing chain in order to assess the validity of the theoretical findings. The step-by-step sketches of the different simulation methodologies are described below.

\section{A. Empirical Approach}

1) Select the parameters $M, N, K, \mu$, and v. 2) Generate channel matrices $\boldsymbol{H}_{\left[l_{0}, l_{0}\right]}$, using $\mathcal{C N}\left(\mu, v^{2}\right)$, and $\boldsymbol{H}_{\left[l_{K}, l_{0}\right]}$, $\boldsymbol{H}_{\left[l_{0}, l_{k}\right]}$, using $\left.\mathcal{C N}(0,1), 3\right)$ Generate complex information vectors $\boldsymbol{s}_{\left[l_{K}, l_{0}\right]}$ and $\boldsymbol{s}_{\left[l_{0}, l_{K}\right]}$. 4) Design linear beamformers $\boldsymbol{V}_{\left[l_{K}, l_{0}\right]}$ and $\boldsymbol{W}_{\left[l_{0}, l_{K}\right]}$ using CSI. 5) Compute the corresponding $\mathcal{I}_{s i_{d}}$.6) Repeat the above process for MC trials. 7) Plot the empirical residual SI distribution.

\section{B. Theoretical Approach}

1-2) Same as above. 3) Generate $\mathcal{I}_{s i_{d}}$ from the Gamma distribution in the Theorem. 4) Repeat for MC trials. 5) Plot the theoretical residual SI distribution.
Without loss of generality, consider a $16 \times 8$ MIMO Rician fading channel with mean $\frac{1}{2}$ and variance 1 . The distributions of the residual SI with linear ZF precoding and decoding are depicted with different number of information streams in the Figure. It can be observed that the theoretical data provides a near exact fit for single-user MIMO (left figure) and a tight approximation for multi-user MIMO (right figure) with respect to the empirical data, respectively. Note that the moment matching accuracy increases for smaller $\mu, v$, and $K$. Increasing $N$ and $M$, for example in the context of massive MIMO, on the other hand, enhances the goodness of the fit.

\section{SUMMARY}

A rigorous study of the residual SI over FD multi-user MIMO Rician fading channels was provided. We considered FD multi-antenna nodes applying linear beamformers to communicate with multiple FD radios. The residual SI fading channels were drawn from a complex Gaussian distribution with arbitrary statistics. We derived the exact first and second positive moments of the residual SI in closedform. The Gamma moment matching approximation was then adopted to develop a unified expression for the residual SI distribution.

\section{APPENDIX}

With Rician fading from $\mathcal{C N}\left(\mu, v^{2}\right), \mathbb{E}\left\{\left|\left\{\boldsymbol{H}_{\left[l_{0}, l_{0}\right]}\right\}_{n, m}\right|^{2}\right\}=$ $\mu^{2}+v^{2}, \mathbb{E}\left\{\left|\left\{\boldsymbol{H}_{\left[l_{0}, l_{0}\right]}\right\}_{n, m}\right|^{4}\right\}=\mu^{2}\left(\mu^{2}+4 v^{2}\right)+2 v^{4}$, and $\mathbb{V}\left\{\left|\left\{\boldsymbol{H}_{\left[l_{0}, l_{0}\right]}\right\}_{n, m}\right|^{2}\right\}=v^{2}\left(2 \mu^{2}+v^{2}\right)$. Considering arbitrary linear precoder/decoder design, we can derive $\mathbb{E}\left\{\left|\left\{\boldsymbol{V}_{\left[l_{K}, l_{0}\right]}\right\}_{m, k}\right|^{2}\right\}=\frac{1}{M}, \mathbb{E}\left\{\left|\left\{\boldsymbol{V}_{\left[l_{K}, l_{0}\right]}\right\}_{m, k}\right|^{4}\right\}=\frac{2}{M(M+1)}$,

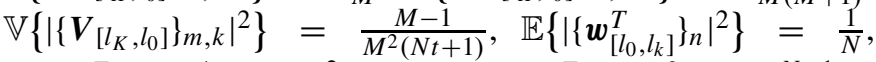
$\mathbb{E}\left\{\left|\left\{\boldsymbol{w}_{\left[l_{0}, l_{k}\right]}^{T}\right\}_{n}\right|^{4}\right\}=\frac{2}{N(N+1)}$, and $\mathbb{V}\left\{\left|\left\{\boldsymbol{w}_{\left[l_{0}, l_{k}\right]}^{T}\right\}_{n}\right|^{2}\right\}=\frac{N-1}{N^{2}(N+1)}$. We use $\delta_{\left[l_{0}, l_{0}\right]} \in \mathcal{C}^{\min (N, M) \times 1}$ to denote the vector containing the $\min (N, M)$ largest singular-values of the residual SI channel at $l_{0}$.

The corresponding first and second positive moments of the residual SI can be respectively developed as in (10) and (11), as shown at the top of the next page. These moments can accordingly be used to derive an expression for the residual SI variance as in (12), as shown at the top of the next page. Using (10) and (12), we can apply the moment matching technique as in the Theorem provided. 

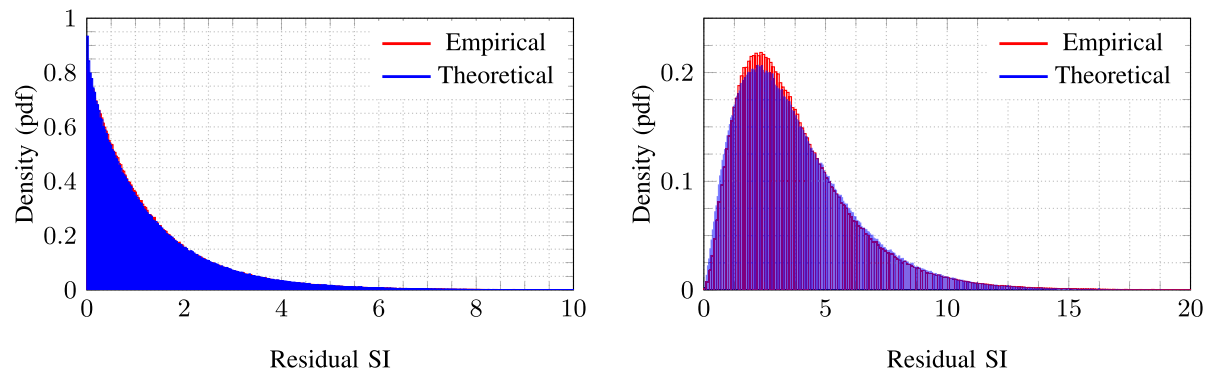

Fig. 1. Simulation parameters are: $M=16, N=8, \mu=\frac{1}{2}, v=1, \mathrm{MC}=10^{7}, K=1$ (left), $K=3$ (right).

$$
\begin{aligned}
\mathbb{E}\left\{\left\|\boldsymbol{w}_{\left[l_{0}, l_{k}\right]}^{T} \boldsymbol{H}_{\left[l_{0}, l_{0}\right]} \boldsymbol{V}_{\left[l_{K}, l_{0}\right]}\right\|^{2}\right\}=\mathbb{E}\left\{\sum_{k=1}^{K}\left|\boldsymbol{w}_{\left[l_{0}, l_{k}\right]}^{T} \boldsymbol{H}_{\left[l_{0}, l_{0}\right]} \boldsymbol{v}_{\left[l_{k}, l_{0}\right]}\right|^{2}\right\} \\
=\mathbb{E}\left\{\sum_{k=1}^{K} \sum_{n_{1}=1}^{N} \sum_{m_{1}=1}^{M} \sum_{n_{2}=1}^{N} \sum_{m_{2}=1}^{M}\left\{\boldsymbol{w}_{\left[l_{0}, l_{k}\right]}^{T}\right\}_{n_{1}}\left\{\boldsymbol{w}_{\left[l_{0}, l_{k}\right]}^{T}\right\}_{n_{2}}^{\dagger}\left\{\boldsymbol{H}_{\left[l_{0}, l_{0}\right]}\right\}_{n_{1}, m_{1}}\left\{\boldsymbol{H}_{\left[l_{0}, l_{0}\right]}\right\}_{n_{2}, m_{2}}^{\dagger}\left\{\boldsymbol{V}_{\left[l_{K}, l_{0}\right]}\right\}_{m_{1}, k}\left\{\boldsymbol{V}_{\left[l_{K}, l_{0}\right]}\right\}_{m_{2}, k}^{\dagger}\right\} \\
=\mathbb{E}\left\{\sum_{k=1}^{K} \sum_{n=1}^{N} \sum_{m=1}^{M}\left|\left\{\boldsymbol{w}_{\left[l_{0}, l_{k}\right]}^{T}\right\}_{n}\right|^{2}\left|\left\{\boldsymbol{H}_{\left[l_{0}, l_{0}\right]}\right\}_{n, m}\right|^{2}\left|\left\{\boldsymbol{V}_{\left[l_{K}, l_{0}\right]}\right\}_{m, k}\right|^{2}\right\}=K\left(\mu^{2}+v^{2}\right)
\end{aligned}
$$

$$
\begin{aligned}
\mathbb{E}\left\{\left\|\boldsymbol{w}_{\left[l_{0}, l_{k}\right]}^{T} \boldsymbol{H}_{\left[l_{0}, l_{0}\right]} \boldsymbol{V}_{\left[l_{K}, l_{0}\right]}\right\|^{4}\right\}=\mathbb{E}\left\{\left(\sum_{k=1}^{K}\left|\boldsymbol{w}_{\left[l_{0}, l_{k}\right]}^{T} \boldsymbol{H}_{\left[l_{0}, l_{0}\right]} \boldsymbol{v}_{\left[l_{k}, l_{0}\right]}\right|^{2}\right)^{2}\right\} \\
=\mathbb{E}\left\{\sum_{k_{1}=1}^{K} \sum_{n_{1}=1}^{N} \sum_{n_{2}=1}^{N} \sum_{m_{1}=1}^{M} \sum_{m_{2}=1}^{M} \sum_{k_{2}=1}^{K} \sum_{n_{3}=1}^{N} \sum_{n_{4}=1}^{N} \sum_{m_{3}=1}^{M} \sum_{m_{4}=1}^{M}\left\{\boldsymbol{w}_{\left[l_{0}, l_{k}\right]}^{T}\right\}_{n_{1}}\left\{\boldsymbol{w}_{\left[l_{0}, l_{k}\right]}^{T}\right\}_{n_{2}}^{\dagger}\left\{\boldsymbol{w}_{\left[l_{0}, l_{k}\right]}^{T}\right\}_{n_{3}}\left\{\boldsymbol{w}_{\left[l_{0}, l_{k}\right]}^{T}\right\}_{n_{4}}^{\dagger}\right.
\end{aligned}
$$

$\left.\times\left\{\boldsymbol{H}_{\left[l_{0}, l_{0}\right]}\right\}_{n_{1}, m_{1}}\left\{\boldsymbol{H}_{\left[l_{0}, l_{0}\right]}\right\}_{n_{2}, m_{2}}^{\dagger}\left\{\boldsymbol{H}_{\left[l_{0}, l_{0}\right]}\right\}_{n_{3}, m_{3}}\left\{\boldsymbol{H}_{\left[l_{0}, l_{0}\right]}\right\}_{n_{4}, m_{4}}^{\dagger}\left\{\boldsymbol{V}_{\left[l_{K}, l_{0}\right]}\right\}_{m_{1}, k_{1}}\left\{\boldsymbol{V}_{\left[l_{K}, l_{0}\right]}\right\}_{m_{2}, k_{1}}^{\dagger}\left\{\boldsymbol{V}_{\left[l_{K}, l_{0}\right]}\right\}_{m_{3}, k_{2}}\left\{\boldsymbol{V}_{\left[l_{K}, l_{0}\right]}\right\}_{m_{4}, k_{2}}^{\dagger}\right\}$

$$
=\mathbb{E}\left\{\sum_{k=1}^{K} \sum_{i=1}^{\min (N, M)}\left|\left\{\boldsymbol{w}_{\left[l_{0}, l_{k}\right]}^{T}\right\}_{i}\right|^{4}\left|\left\{\boldsymbol{\delta}_{\left[l_{0}, l_{0}\right]}\right\}_{i}\right|^{4}\left|\left\{\boldsymbol{V}_{\left[l_{K}, l_{0}\right]}\right\}_{i, k}\right|^{4}\right\}
$$

$+2 \mathbb{E}\left\{\left.\left.\sum_{k=1}^{K} \sum_{i_{1}=1, i_{1} \neq i_{2}}^{\min (N, M)} \sum_{i_{2}=1, i_{2} \neq i_{1}}^{\min (N, M)}\left|\left\{\boldsymbol{w}_{\left[l_{0}, l_{k}\right]}^{T}\right\}_{i_{1}}\right|^{2}\left\{\boldsymbol{w}_{\left[l_{0}, l_{k}\right]}^{T}\right\}_{i_{2}}\right|^{2}\left|\left\{\boldsymbol{\delta}_{\left[l_{0}, l_{0}\right]}\right\}_{i_{1}}\right|^{2}\left|\left\{\boldsymbol{\delta}_{\left[l_{0}, l_{0}\right]}\right\}_{i_{2}}\right|^{2}\left\{\boldsymbol{V}_{\left[l_{K}, l_{0}\right]}\right\}_{i_{1}, k}\right|^{2}\left|\left\{\boldsymbol{V}_{\left[l_{K}, l_{0}\right]}\right\}_{i_{2}, k}\right|^{2}\right\}$

$+2 \mathbb{E}\left\{\sum_{k_{1}=1}^{K-1} \sum_{k_{2}=k_{1}+1}^{K}\left|\sum_{n=1}^{N} \sum_{m=1}^{M}\left\{\boldsymbol{w}_{\left[l_{0}, l_{k}\right]}^{T}\right\}_{n}\left\{\boldsymbol{H}_{\left[l_{0}, l_{0}\right]}\right\}_{n, m}\left\{\boldsymbol{V}_{\left[l_{K}, l_{0}\right]}\right\}_{m, k_{1}}\right|^{2}\left|\sum_{n=1}^{N} \sum_{m=1}^{M}\left\{\boldsymbol{w}_{\left[l_{0}, l_{k}\right]}^{T}\right\}_{n}\left\{\boldsymbol{H}_{\left[l_{0}, l_{0}\right]}\right\}_{n, m}\left\{\boldsymbol{V}_{\left[l_{K}, l_{0}\right]}\right\}_{m, k_{2}}\right|^{2}\right\}$

$$
=\frac{4 K}{(N+1)(M+1)}\left(N M \mu^{4}+(N+M) v^{2}\left(2 \mu^{2}+v^{2}\right)\right)+\frac{2 K(N-1)(M-1)}{(N+1)(M+1)} v^{2}\left(2 \mu^{2}+v^{2}\right)
$$

$+K(K-1)\left(\frac{1}{M-K+2}+1\right)\left(\frac{2 N M}{N M+N+M+1} \mu^{4}+v^{2}\left(2 \mu^{2}+v^{2}\right)\right)=K\left(\frac{M+1}{M-K+2}+K\right)\left(\frac{2 N M}{N M+N+M+1} \mu^{4}+v^{2}\left(2 \mu^{2}+v^{2}\right)\right)$

$$
\begin{aligned}
\mathbb{V}\left\{\left\|\boldsymbol{w}_{\left[l_{0}, l_{k}\right]}^{T} \boldsymbol{H}_{\left[l_{0}, l_{0}\right]} \boldsymbol{V}_{\left[l_{K}, l_{0}\right]}\right\|^{2}\right\}=\mathbb{E}\left\{\left\|\boldsymbol{w}_{\left[l_{0}, l_{k}\right]}^{T} \boldsymbol{H}_{\left[l_{0}, l_{0}\right]} \boldsymbol{V}_{\left[l_{K}, l_{0}\right]}\right\|^{4}\right\}-\mathbb{E}\left\{\left\|\boldsymbol{w}_{\left[l_{0}, l_{k}\right]}^{T} \boldsymbol{H}_{\left[l_{0}, l_{0}\right]} \boldsymbol{V}_{\left[l_{K}, l_{0}\right]}\right\|^{2}\right\}^{2} \\
=\frac{K}{M-K+2}\left(\frac{K(M-K+2)(N M-N-M-1)+2 N M(M+1)}{(N+1)(M+1)} \mu^{4}+(M+1) v^{2}\left(2 \mu^{2}+v^{2}\right)\right)
\end{aligned}
$$

\section{REFERENCES}

[1] M. Duarte, C. Dick, and A. Sabharwal, "Experiment-driven characterization of full-duplex wireless systems," IEEE Trans. Wireless Commun., vol. 11, no. 12, pp. 4296-4307, Dec. 2012.

[2] D. Bharadia and S. Katti, "Full duplex MIMO radios," in Proc. USENIX Symp. Netw. Syst. Design Implement., 2014, pp. 1-13.

[3] G. Zheng, "Joint beamforming optimization and power control for fullduplex MIMO two-way relay channel," IEEE Trans. Signal Process., vol. 63, no. 3, pp. 555-566, Feb. 2015.
[4] D. Nguyen, L.-N. Tran, P. Pirinen, and M. Latva-Aho, "On the spectral efficiency of full-duplex small cell wireless systems," IEEE Trans. Wireless Commun., vol. 13, no. 9, pp. 4896-4910, Sep. 2014.

[5] I. Atzeni and M. Kountouris, "Full-duplex MIMO small-cell networks: Performance analysis," in Proc. IEEE Global Commun. Conf., Dec. 2015, pp. 1-6.

[6] A. Shojaeifard, K. A. Hamdi, E. Alsusa, D. K. C. So, J. Tang, and K.-K. Wong, "Design, modeling, and performance analysis of multiantenna heterogeneous cellular networks," IEEE Trans. Commun., vol. 64, no. 7, pp. 3104-3118, Jul. 2016. 